\title{
Infochemical-mediated preference behavior of the maize weevil, Sitophilus zeamais Motschulsky, when searching for its hosts
}

\author{
Qingfeng Tang, Yuejin Wu*, Binmei Liu \& Zengliang Yu
}

Tang, Q., Wu, Y., Liu, B. \& Yu, Z. 2008: Infochemical-mediated preference behavior of the maize weevil, Sitophilus zeamais Motschulsky, when searching for its hosts. - Entomol. Fennica 19: 257-267.

Behavioral responses of $S$. zeamais to odours from pulverized wheat, brown rice, sorghum, buckwheat, peanut and cork were compared in a Y-tube-olfactometerbioassay. Results showed that both sexes responded to host volatiles and males were more sensitive than females. The strongest responses to grains of wheat and brown rice were found, and insects reared on these were dramatically heavier than on other tested materials (grain sorghum, buckwheat, peanut and cork). Multiple-choices tests, in which volatiles from males that were removed from the wheat within different minutes were simultaneously presented in the chamber, were used to study whether males can release aggregation pheromone if not on the grain. The pheromone was released by males within about 13 minutes after removal from the grains. Responses to pheromones produced by males were skewed toward females although both sexes were attracted. Both sexes responded most strongly to the odour source comprising pheromone with host volatiles.

Q. Tang, Y. Wu*, B. Liu \& Z. Yu, Key Laboratory of Ion Beam Bioengineering, Institute of Plasma Physics, Chinese Academy of Sciences, Hefei, China; *E-mail of the corresponding author: yjwu@ipp.ac.cn

Received 8 May 2008, accepted 16 September 2008

\section{Introduction}

The maize weevil (Sitophilus zeamais Motschulsky; Coleoptera: Curculionidae) one of the most common and destructive storage pest in the world (Hill 1983, Haines 1991), and an internal feeder whose adults attack whole kernels and whose larvae feed and develop entirely within kernels (Storey 1987). Damage caused by $S$. zeamais stored cereal crops may be extremely high. Infestation may start in the mature crop standing in the field and continue in the granary where considerable losses can occur (Giles 1969; Haines 1991). This post-harvest insect contributes to the deterioration of the quality and the quantity of stored grain (Kouninki et al. 2007). Currently, the primary means of maize weevil control in warm climates is the use of fumigants and residual chemical insecticides (Faruki et al. 2005, \& Moharramipour 2007). Synthetic chemical pesticides are usually applied by the farmers to reduce losses during storage (Adane et al. 1996). In recent years, however, the overreliance and the use of chemical insecticides in crop pest control programs (around the world) has resulted in environmental damage, pest resurgence, pest resistance to insecticides, and lethal effects on non-target organisms. Furthermore, because of cost, these pesticides are becoming increasingly inaccessible to farmers, particularly in develop- 
ing countries. This fact, combined with the consumer's demand for residue-free food, prompted researchers to evaluate other alternative reducedrisk control methods for stored-grain protection. These methods include, among others, the application of chemical ecology in the control of stored-grain insects. of semiochemical-based pest management systems in stored-products is being necessitated by the withdrawal of approval for use of many synthetic insecticides and the fumigant methyl bromide.

Infochemical cues are generally considered to play a pivotal role in the location, evaluation and utilization of hosts by herbivorous insects (Francis et al. 2005). Numerous species of insects have been shown to be attracted to (single or blends of) volatiles of their host plants (Visser 1986). The response of maize weevil to olfactory stimuli has been demonstrated earlier and food-related odours may contain potential attractants (Honda \& Oshawa 1990, Ben \& Xuan 1992, Pike et al. 1994). However, it is not clear whether $S$. zeamais is capable of using olfactory cues to discriminate between volatiles from suitable and unsuitable plant species during the host-finding process.

In addition to the role of plant volatiles as attractants, pheromones promote aggregation of $S$. zeamais on located food sources. The aggregation pheromone 'Sitophilure' (4S, 5R)-5-hydroxy-4-methyl-3-heptanone is produced by male beetles. Investigation of pheromone trapping has been restricted to laboratory studies on walking Sitophilus spp. (Walgenbach et al. 1987, Trematerra \& Girgenti 1989) and a preliminary field study in Kenya on trapping both flying and walking beetles (Hodges \& Dobson 1998). However, the effect of combining host volatiles and aggregation pheromone on the behavioural responses of $S$. zeamais adults has not been determined. Previous reports deem the aggregation pheromones by stored product pests can be stopped very quickly within minutes when males are not on suitable breeding substrate (Bashir et al. 2001, Plarre 1998). However, little is known about the release of pheromones from males that were removed from the host within different minutes.

An understanding of the responses ofto cues employed in the host-finding process may be of practical value in improving semiochemicalbased trapping system for the species. We expect that semiochemical-based methods will become important components of integrated pest management (IPM) of various commodity systems.

In this study, there were three objectives. The first was to confirm the orientation of $S$. zeamais towards plant volatiles as cues for the purpose of selecting plant species that are suitable hosts and to examine whether the orientation is related to performance of the insects. The second was to determine whether males release aggregation pheromones if not on the grain. The third was to investigate the response of the species to a combination of male-produced aggregation pheromone and host odours.

\section{Material and methods}

\subsection{Insects and substrate}

The $S$. zeamais used were originally collected in the Province of Henan (China) and subsequently cultured in the laboratory on whole maize grain at $13.5 \%$ moisture content. Intact whole grain of uniform size were chosen for the experiment and equilibrated in a constant temperature and humidity $(\mathrm{CTH})$ room at $28 \pm 1{ }^{\circ} \mathrm{C}$ and $70 \pm 5 \%$ r.h., and under a $8: 16 \mathrm{~h}$ (light : dark) photoperiod. All of the experiments were carried out in the same CTH room. Weevils used were obtained from laboratory stocks, and the culturing techniques used have been described by Walgenbach et al. (1983). Insects used were generally 0-7 days post adult eclosion except where stated otherwise. Insects were removed from culture and held in clean glass tubes without food at the test conditions for $48 \mathrm{~h}$ prior to behavioural bioassays. Dimorphic rostral characteristics were used to separate the sexes (Halstead 1963).

\subsection{Plant materials}

Tissues of six plant species were used in the experiments including wheat grain, brown rice grain, sorghum grain, buckwheat grain, peanut grain, which have been reported as possible hosts of S. zeamais, and cork, reported as a non-host of it. Freshly harvested grain of winter wheat, brown rice and grain sorghum were obtained 
from farms located in Hefei, Anhui, China. Unprocessed shelled buckwheat, peanut grain and cork were obtained from a commercial grocery store. To kill any insects in kernels, the plant materials were deep frozen within the bags until required for the experiments. Moisture content of plant materials used in this and subsequent experiments in the study ranged from $12.6 \%$ to $14.1 \%$ at the start of the experiment.

\subsection{Experiment 1: Response to plant volatiles}

Volatiles from wheat grain, brown rice grain, sorghum grain, buckwheat grain, peanut grain and cork were used to compare the responses of male and female $S$. zeamais. To ensure plant tissues used as a source of volatiles were of similar size, plant materials were pulverized using a mechanical grinder and sifted using a $\lesssim 21$ sieve. Evaluations were performed in a constant temperature and humidity $(\mathrm{CTH})$ room at $28 \pm 1{ }^{\circ} \mathrm{C}$ and $70 \pm 5 \%$ r.h., under dim light supplied with a red fluorescent tube.

A Y-tube olfactometer was used to determine the attractiveness of the various potential plant hosts tested against clean air. A detailed description of the Y-tube olfactometer is given by Takabayashi and Dicke (1992). In our experiments two Erlenmeyer flasks were used as odour chambers. About $200 \mathrm{~g}$ of the materials to be tested were weighed into one of the flasks, while the other flask remained empty when clean air was used as a control. Using teflon tubing, each of the odour chambers was connected to one arm of the olfactometer. The flasks were kept behind a cloth screen to rule out visual perception of the grain. Airflow through both arms was generated by air pressure, and purified by use of a charcoalfilled gas wash bottle. The airflow through the arms of the Y-tube $(280 \mathrm{ml} / \mathrm{min})$ was adjusted by flow meters which were positioned between flasks and the Y-tube. The flowmeters were cleaned with ethanol each time the test plant was changed.

One hundred beetles (50 males and 50 females) were tested individually per treatment. Responses of 50 males and 50 females, tested in alternate batches of five, were observed for each of the odour sources. A single adult of S. zeamais was introduced into the Y-olfactometer at the entrance of the stem, with maximum observation duration of $20 \mathrm{~min}$ per beetle and the responses were considered positive when the insects reached at least $3 \mathrm{~cm}$ along the arm connected to the test chambers. Insects were used only once and discarded. Additionally connections of the odour sources to the arms were reversed after testing five insects to avoid asymmetrical bias. After every 10 beetles the Y-tube was cleaned with ethanol and the teflon tubes were connected to the Y-tube the other way around. The beetles were always tested between 9:00 and 17:00 h.

For each test, we determined 'Activity' and 'Preference' (choice) of the weevils. Activity was measured as the number of the weevils that showed behavioral activity in the olfactometer (they were trapped in the left or right arm of the Ytube) compared to weevils not responding (not entering the left or right arm and remaining in the common tube of the Y-tube). Preference was measured as the number of active weevils choosing one or the other side in the olfactometer.

\subsection{Experiment 2: Progeny production}

No-choice assays were performed to determine how wheat grain, brown rice grain, sorghum grain, buckwheat grain, peanut grain and cork affect the development and reproductive success of S. zeamais.

The original Dobie method (Dobie 1974) was used in this study. Five replicates of $32 \pm 0.3 \mathrm{~g}$ per test plant species were weighed into round plastic pots $(67 \times 32 \mathrm{~mm})$ with screw-top lids. Adult, $0 \pm 7$ day-old $S$. zeamais were sexed by their rostral characteristics (Halstead 1963) and an appropriate number placed on each of the test plant species for seven days. This conditioning period was to eliminate any possible short-term behavioral changes associated with an altered environment. After this period, ten female and ten male 7-14 day-old adults were placed on the test plant species for 7 days. On day 7, all the insects were sieved off. Each pot was checked regularly before the expected time of emergence of the first filial (F1) generation. The date of the first emerging F1 was noted and the number of F1 individuals 
emerging from each pot was recorded daily during the working week until all the F1 adults had emerged. Taking into consideration that mating in S. zeamais does not occur before weevils are $3 \mathrm{~d}$ old (Walgenbach \& Burkholder 1987), there was little possibility of third generation adults emerging (Povey \& Sibly 1992). The criteria for determining the end of the F1 generation was the absence of emergence for at least five consecutive days, excluding the first week of emergence. Fresh body weight of emerged male and female beetles from different treatments was determined to the nearest $0.0001 \mathrm{~g}$, using a Sartorius electronic microbalance type CP124S.

Susceptibility indices were used as a measure of the susceptibility of test plant species to infestation by $S$. zeamais. The higher the index, the greater the susceptibility of the test plant species (Gudrups et al. 2001). These indices are defined by the following formula:

Dobie Index: D.I. $=\frac{\operatorname{LnF} \times 100}{D M E}$

where D.I. is the susceptibility index, $L n$ the natural logarithm, $F$ the total number of $F 1$ adults, and $D M E$ the date of median emergence of $\mathrm{F} 1$ (days).

\subsection{Experiment 3: Do males release pheromones if not on the grain?}

To study whether males can release aggregation pheromone if not on the grain, the responses of weevils to males that were removed from the wheat within different minutes were measured using a four-arm olfactometer similar to the one described by Vet et al. (1983), with modifications to accommodate the size $(\sim 3 \mathrm{~mm})$ of $S$. zeamais. The arena of the olfactometer was made of three parts: a $140 \times 140 \times 5 \mathrm{~mm}$ clear acrylic square (top), a $140 \times 140 \times 5 \mathrm{~mm}$ white acrylic square with a four-pointed star-shape cutout exposure chamber $\left(90^{\circ}\right.$ arc, radius $\left.80 \mathrm{~mm}\right)$, and a $200 \times 200$ $\mathrm{mm}$ white teflon square (base). The top was cemented to the exposure chamber, and two plastic clamps were used to hold it firmly to the base. Each olfactometer arm was divided into three regions: the "release" region at the center, the "visit" region nearest to the center, and the "selection" region nearest to the odour source. Evalua- tions were performed in a constant temperature and humidity $(\mathrm{CTH})$ room at $28 \pm 1{ }^{\circ} \mathrm{C}$ and $70 \pm 5 \%$ r.h., under dim light supplied with a red fluorescent tube with no natural lighting.

The airflow $(100 \mathrm{ml} / \mathrm{min} / \mathrm{arm})$ inside the arena was equalized using one flowmeter at each arm and a terminal flowmeter between the arena and the pump. Charcoal-filtered air was passed through treatment chambers that enclosed the odour sources inside a glass flask, and tubing leading from each of the odours converged into the olfactometer arena. Behavioral data were visually recorded using a stopwatch. Individual $S$. zeamais were used only once.

A single test beetle was released into a central hole of the chamber through the central hole in the glass-plate cover using a fine brush. To increase the chances that beetle response was due to choice of a zone rather than movement caused by disturbance/handling, any beetles not spending $10 \mathrm{~s}$ in the centre before moving into an odour zone were not counted. Each beetle was observed for $5 \mathrm{~min}$ in the chamber. The time spent by the beetle in different zones was recorded. However, if a beetle entered one of the four air-delivery pipes, the test was terminated for that insect and the remaining time was awarded to that particular zone. The time spent by each beetle in each of the four odour zones was recorded to determine the preference of the beetles for different odour/volatile sources.

Three different odour sources were used to compare the responses of adult male and female S. zeamais; (1) 100 males within 3 8 minutes after removal from the wheat, (2) 100 males within $8 \sim 13$ minutes after removal from the wheat, and (3) 100 males within 13 18 minutes after removal from the wheat.

For the multiple-choice tests, volatiles of three odour sources were presented in the exposure chamber at the same time, one from each airdelivery pipe, with clean air from the fourth pipe. Responses of ten males and ten females were observed. The whole procedure was repeated five times. In this way the responses of a total of 100 beetles (50 males and 50 females) to five replications of each odour source were observed during the experiment. The experimental design of these multiple-choice tests was strengthened both by replicating the odour sources and by interchang- 


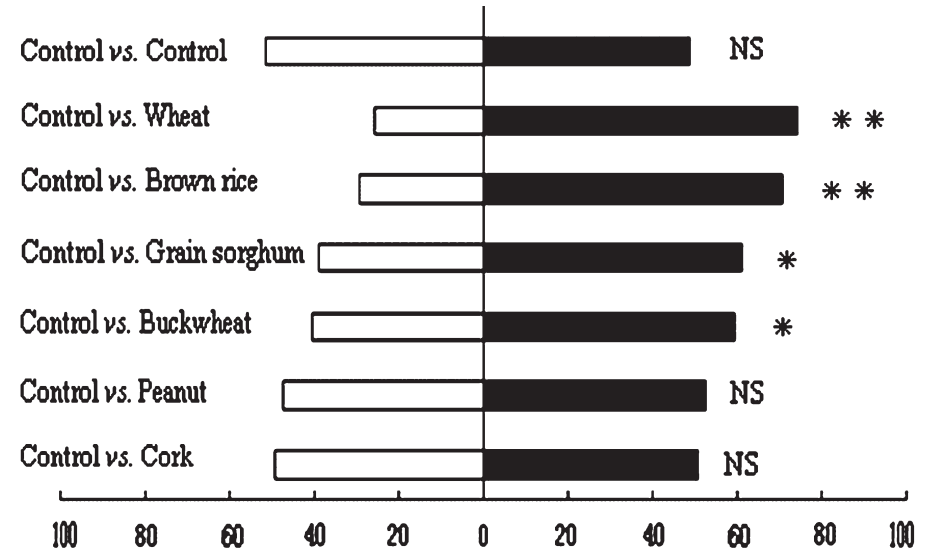

Preference of responding (active) male weevils (\%)

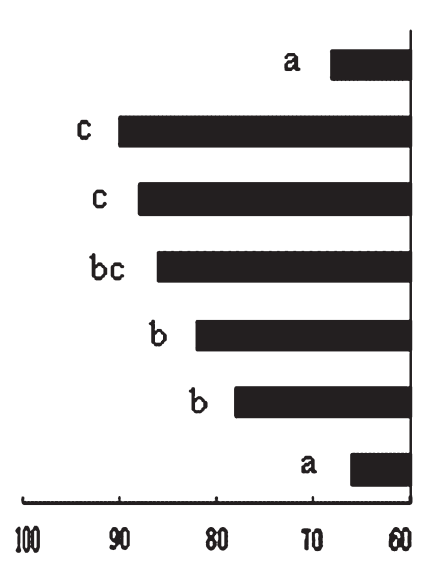

Male weevils responding (\%)

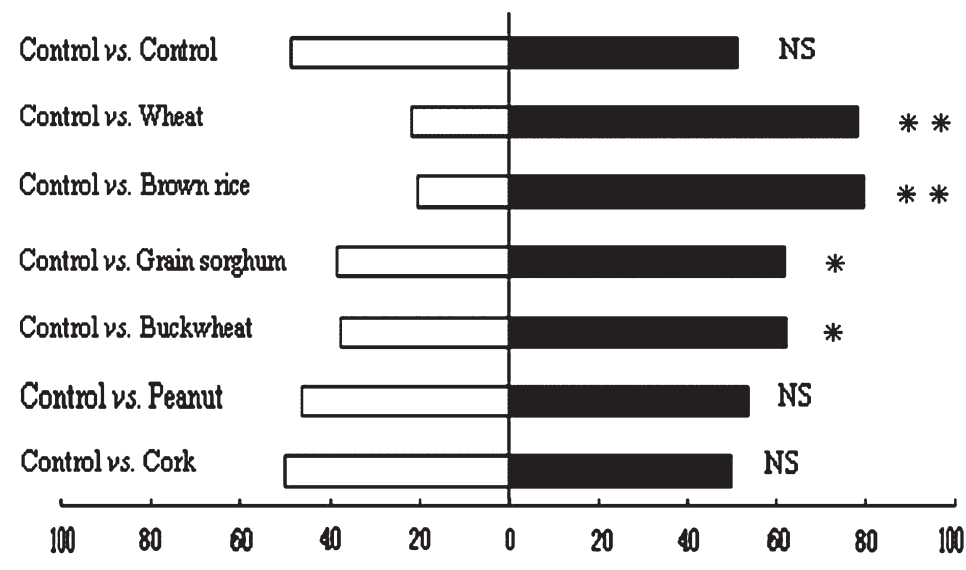

Preference of responding (active) female weevils (\%)

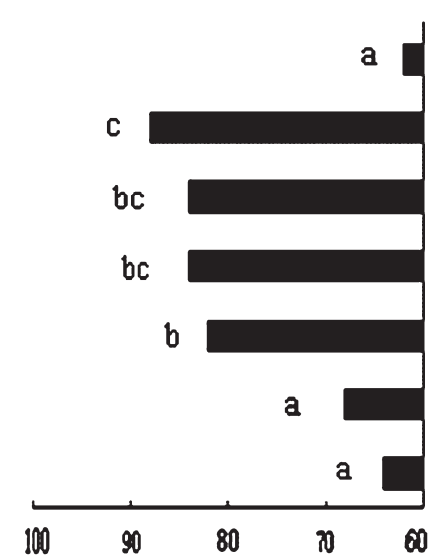

Female weevils responding (\%)

Fig. 1. Preference and activity of $S$. zeamais in a Y-tube olfactometer to odours from six plant species. Asterisks indicate significant differences from even distribution at ${ }^{*} P<0.05$, or ${ }^{* *} P<0.01$. Activity is expressed as percentage weevils responding, and bars followed by different letters indicate significantly different activity of the weevils between the treatments $(P<0.05)$. The mean values shown are predicted values from regression analysis. NS = not significantly different.

ing males to avoid male variation in pheromone output influencing the results.

In the multiple tests, the air-delivery pipes at which the volatiles or clean air were presented were initially assigned randomly to different odour sources. After one quarter of the olfactometer tests was completed, the order in which the pipes were connected to the exposure chamber was changed so that during the experiment each zone of the test arena received volatiles from each source. After testing every ten insects, the deliv- ery pipes and bioassay chamber were cleaned. The beetles were always tested between 9:00 and 17:00 h.

\subsection{Experiment 4: Response to host odour and aggregation pheromone interaction}

The responses of weevils to host odour and aggregation pheromone interaction were measured using a four-arm olfactometer. Three different 
odour sources were used to compare the responses of adult male and female $S$. zeamais; (1) host volatiles emanating from $500 \mathrm{~g}$ of whole, sound, grain of wheat (wheat volatiles alone), (2) 100 pheromone-releasing males within 3 minutes after removal from the wheat, and (3) 100 males placed on the surface of the $500 \mathrm{~g}$ of wheat grain from where they were easily retrieved at the end of the test (wheat-volatiles blend aggregation pheromone). The remaining details were the same as in experiment 3 .

\subsection{Statistical analysis}

For the Y-tube choice experiments a $\chi^{2}$ test (oneway) for goodness-of-fit with one degree of freedom was used to determine significance.

Data from the Experiment 2 were analyzed using SAS PROC MIXED (SAS Institute, 2001). Before data analysis, count data were transformed using the $\log (\mathrm{x}+1)$, in order to satisfy the assumptions of normality and homogeneity of variance (Zar 1999). Tukey's Studentized range test was used to separate means (Tukey 1953). Data on progeny production and development time on different plant tissues were analyzed as a one-factor experiment, and those for weight of beetles emerged as a two-factor experiment in which the main factors were plant species and beetle sex.

Data from the Experiment 3 and Experiment 4 were analysed by a Friedman test for K-related samples, which was used to compare responses in all four zones. When the Friedman test showed a significant difference, a Wilcoxon test (for 2-related samples) was used for pairwise comparisons between sources. The responses of males and females were compared using a Mann-Whitney test for each of the three odour/volatile sources and control.

\section{Results}

\subsection{Responses of Sitophilus zeamais to volatiles from different plant species}

Of the host plants (wheat grain, brown rice grain, grain sorghum, buckwheat grain and peanut grain) and non-host (cork) tested (Fig. 1), odour from wheat grain, brown rice grain, sorghum grain and buckwheat grain stimulated significantly more male and female weevils to become active than the control treatment. Significant differences in male weevils' activity were also found between the peanut grain treatment and the control treatment $\left(\chi^{2}=4.08\right.$, d.f. $\left.=1, P=0.043\right)$, but the percentage of responding female weevils under the peanut grain treatment and the control treatment did not differ significantly $\left(\chi^{2}=0.83\right.$, d.f. $=1, P=0.362)$. The activity in tests with nonhost (cork) treatment did not differ from the control treatment (females: $2=0.72$, d.f. $=1, P=$ 0.396; males: $2=0.58$, d.f. $=1, P=0.446$ ).

$S$. zeamais showed no statistically significant difference (females: $\chi^{2}=2.67$, d.f. $=1, P=0.102$; males: $\chi^{2}=1.56$, d.f. $\left.=1, P=0.211\right)$ in choice between the two arms of the Y-tube olfactometer when clean air was passed through both the left and right arm. This result confirmed that the weevils' behaviour within the olfactometer was unbiased.

The active female and male weevils were strongly attracted to wheat $\left(78.1 \%\right.$ preference, $\chi^{2}$ $=12.51$, d.f. $=1, P=0.0004$, and $74.2 \%$ preference, $\chi^{2}=11.91$, d.f. $=1, P=0.0005$, respectively), brown rice $\left(79.6 \%\right.$ preference, $\chi^{2}=10.73$, d.f. $=1, P=0.001$, and $70.8 \%$ preference, $\chi^{2}=$ 9.52 , d.f. $=1, P=0.002$, respectively), grain sorghum $\left(61.7 \%\right.$ preference, $\chi^{2}=4.47$, d.f. $=1, P=$ 0.034 , and $61.1 \%$ preference, $\chi^{2}=5.81$, d.f. $=1, P$ $=0.016$, respectively) and buckwheat $(62.3 \%$ preference, $\chi^{2}=4.96$, d.f. $=1, P=0.026$, and $59.4 \%$ preference, $\chi^{2}=6.32$, d.f. $=1, P=0.012$, respectively), whereas there was no preference for peanut $\left(53.7 \%\right.$ preference, $\chi^{2}=1.07$, d.f. $=1$, $P=0.301$, and $52.6 \%$ preference, $\chi^{2}=0.89$, d.f. $=$ $1, P=0.345$, respectively) or cork (49.7\% preference, $\chi^{2}=0.77$, d.f. $=1, P=0.380$, and $50.6 \%$ preference, $\chi^{2}=0.79$, d.f. $=1, P=0.374$, respectively).

\subsection{Development assays}

The results in Table 1 and Fig. 2 show that each of the six plant tissues affected the development and reproductive success of $S$. zeamais. $S$. zeamais were still alive in wheat grain, brown rice grain, 
Table 1. Reproductive success (means $\pm \mathrm{SE}$ ) of $S$. zeamais reared on six different plant species.

\begin{tabular}{llllll}
\hline Plant species & $\begin{array}{l}\text { Number of } \\
\text { emerged adults }\end{array}$ & $\begin{array}{l}\text { Development } \\
\text { time (days) }\end{array}$ & $\begin{array}{l}\text { Weight of } \\
\text { males }(\mathrm{mg})\end{array}$ & $\begin{array}{l}\text { Weight of } \\
\text { females (mg) }\end{array}$ & $\begin{array}{l}\text { Weight of } \\
\text { adults (mg) }\end{array}$ \\
\hline Wheat & $54.0 \pm 11.2 \mathrm{a}$ & $32.2 \pm 2.0 \mathrm{c}$ & $1.6 \pm 0.2$ & $1.8 \pm 0.2$ & $1.7 \pm 0.2 \mathrm{a}$ \\
Brown rice & $52.4 \pm 12.3 \mathrm{a}$ & $34.0 \pm 0.8 \mathrm{c}$ & $1.6 \pm 0.3$ & $1.6 \pm 0.4$ & $1.6 \pm 0.3 \mathrm{a}$ \\
Grain sorghum & $30.6 \pm 9.8 \mathrm{~b}$ & $39.8 \pm 2.6 \mathrm{~b}$ & $1.2 \pm 0.2$ & $1.3 \pm 0.2$ & $1.2 \pm 0.2 \mathrm{~b}$ \\
Buckwheat & $28.0 \pm 13.1 \mathrm{~b}$ & $39.4 \pm 1.8 \mathrm{~b}$ & $1.2 \pm 0.3$ & $1.3 \pm 0.2$ & $1.2 \pm 0.2 \mathrm{~b}$ \\
Peanut & $7.2 \pm 9.4 \mathrm{c}$ & $45.4 \pm 3.4 \mathrm{a}$ & $0.9 \pm 0.4$ & $1.1 \pm 0.2$ & $1.0 \pm 0.2 \mathrm{~b}$ \\
Cork & 0 & - & - & - & - \\
\hline
\end{tabular}

Means within columns followed by different letters are significantly different at $P<0.05$.

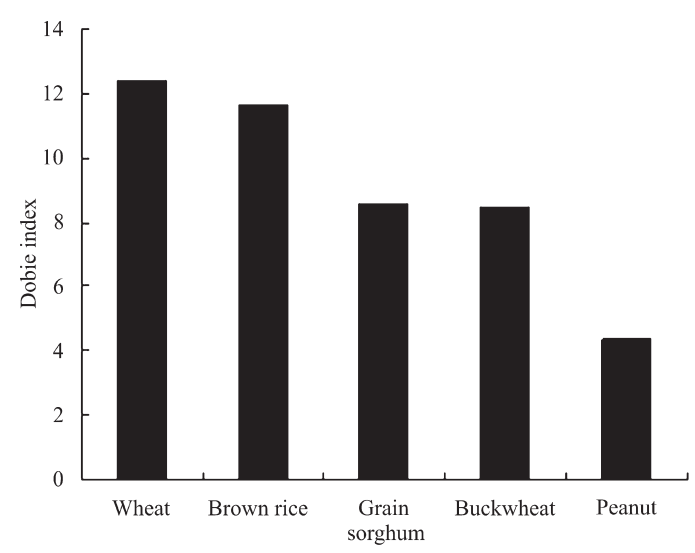

Fig. 2. Dobie index (D.I.) of susceptibility of six different plant species to $S$. zeamais. The higher the index, the greater the susceptibility level.

sorghum grain, buckwheat grain, peanut grain, which have been reported as possible hosts of $S$. zeamais; however, no reproduction occurred at all on cork, reported previously as a non-host.

Significantly higher reproduction was observed on wheat or brown rice than on grain sorghum, buckwheat or peanut. The lowest number of progeny and the longest development periods are associated with peanuts. There were no significant differences between the mean weights of emerged male or female $S$. zeamais within plant species (Table 1). However, the main effect of plant species was significant. Mean weights of beetles raised on wheat or brown rice were heavier than those reared on other plant species (Table 1). Weight of emerged S. zeamais on grain sorghum, buckwheat and peanut were similar. The Dobie index increased in the order of peanut, buckwheat, grain sorghum, brown rice, wheat (Fig. 2).

\subsection{Response to volatiles from males that were removed from the wheat within different minutes}

Amale and female $S$. zeamais more time in the zones receiving volatiles of 100 males (removed from the wheat within 3 8 minutes), or 100 males (removed from the wheat within $8 \sim 13$ minutes) than in the zone receiving the clean air control sources (Table 2). Based in percentage of time spent in each zone, the analysis of the data showed that difference between treatment combinations were statistically significant except in the case of males between 100 males (removed from the wheat within 8 13 minutes) and 100 males (removed from the wheat within 13 18 minutes) $(F 3,196=0.78, P=0.757)$, and for both sexes between 100 males (removed from the wheat within 13 18 minutes) and the clean air control (males:

Table 2. Mean percentage ( \pm SE) of time (of the total 5 min) spent by males and females of $S$. zeamais in each odour zone in multiple-choice tests.

\begin{tabular}{lll}
\hline $\begin{array}{l}\text { Odour sources } \\
\text { (within minutes after } \\
\text { removal from the wheat) }\end{array}$ & Males & Females \\
\hline 100 males & & \\
$\quad$ (within 3-8 minutes) & $38.7 \pm 5.2 \mathrm{a}$ & $52.3 \pm 4.7 \mathrm{a}$ \\
100 males & & \\
$\quad$ (within 8-13 minutes) & $23.6 \pm 3.3 \mathrm{~b}$ & $37.2 \pm 8.4 \mathrm{~b}$ \\
100 males & & \\
$\quad$ (within 13-18 minutes) & $20.2 \pm 7.2 \mathrm{bc}$ & $5.9 \pm 3.3 \mathrm{c}$ \\
Control & $17.6 \pm 3.1 \mathrm{c}$ & $4.7 \pm 2.9 \mathrm{c}$ \\
\hline
\end{tabular}

Means within columns followed by different letters indicate significant difference at $P<0.05$ between the odour source treatments. 
$F 3,196=1.58, P=0.342$; females: $F 3,196=$ $2.55, P=0.187)$.

\subsection{Response to host odour and aggregation pheromone interaction}

In the four arm olfactometer, adult male and female $S$. zeamais significantly longer (males: $F 3,196=8.58, P=0.023$; females: $F 3,196=$ 9.75, $P=0.017$ )in the zones receiving odour sources than receiving clean air (Table 3). Furthermore, both males and females spent more time in the zone receiving the mixture of wheat volatiles and aggregation pheromone than in either wheat odour or aggregation pheromone alone. Females responded more strongly to aggregation pheromone alone than to the odour of wheat $(F 3,196=8.78, P=0.024)$ and when compared to males, they responded more strongly to the mixture of wheat odours and aggregation pheromone $(F 3,196=9.04, P=0.016)$. In contrast, males showed a stronger response than females to wheat odours alone $(F 3,196=8.65, P=$ $0.041)$. There was no significant difference between males and females in response to the aggregation pheromone alone $(F 3,196=0.91, P=$ 0.382 ) although more females were attracted to this.

\section{Discussion}

\subsection{Role of plant odour in host finding and acceptance by Sitophilus zeamais}

Both sexes of $S$. zeamais responded positively to volatiles emitted from the host plant species tested, while not from the non-host tested, possibly because these odours indicate feeding and/or oviposition sites. Our results from experiment 1 indicated that the host plant species tested had volatile constituents that were attractive to $S$. zeamais. Based on the responses of $S$. zeamaisto different hosts, it is possible that the same or similar attractive compounds may be found in plant products that are taxonomically different. Although $S$. zeamais was able to feed on peanut, the fact that $S$. zeamais was not attracted to peanut seeds and unable to distinguish between peanut
Table 3. Mean ( \pm SE) percentage of time (of the total 5 min) spent by males and females of $S$. zeamais in each odour zone in multiple-choice tests.

\begin{tabular}{lll}
\hline Odour sources & Males & Females \\
\hline Wheat $(500 \mathrm{~g})$ & $26.2 \pm 6.8 \mathrm{~b}$ & $7.9 \pm 4.2 \mathrm{c}^{* *}$ \\
100 males & $15.4 \pm 4.3 \mathrm{c}$ & $19.2 \pm 3.6 \mathrm{~b}$ \\
Wheat $(500 \mathrm{~g})+100$ males & $55.1 \pm 8.2 \mathrm{a}$ & $71.3 \pm 7.9 \mathrm{a}^{*}$ \\
Control & $3.4 \pm 1.6 \mathrm{~d}$ & $1.5 \pm 0.8 \mathrm{~d}$ \\
\hline
\end{tabular}

Asterisks indicate significant differences from even distribution at ${ }^{*} P<0.05$, or ${ }^{* *} P<0.01$. Means followed by different letters indicate significant difference at $P<0.05$ between the odour source treatments.

and clear air in the bioassay might be due to the low content of signals in peanut that being below the detecting limit of maize weevil, or the high content of volatiles from high oil content in peanut.

Results from experiment 2 showed that the most suitable foods were wheat and brown rice among the plant species tested. $S$. zeamais was mostly sensitive to volatiles from wheat and brown rice. These two grains were judged to be the most suitable hosts based on the greater reproduction and heavier weights of emerged beetles relative to other plant species tested.

Our findings suggested that $S$. zeamais is capable of using olfactory cues to discriminate between volatiles from suitable and unsuitable plant species during the host-finding process. Since a high cost in the form of mortality to adults or wasted oviposition attempts may result from choosing the wrong host plant, it is possible that $S$. zeamais feeding on stored grain have evolved to respond to olfactory cues indicative of a suitable host, to avoid costly feeding or oviposition on non-host. The use of host-associated volatiles to discriminate between host odours has been demonstrated for a range of coleopteran species (Byers 1995, Pureswaran \& Borden 2005, Edde \& Phillips 2006).

\subsection{Evidence for the release of aggregation pheromone by $S$. zeamais males after removal from the grains}

A majority of expert take it for granted that aggregation pheromones of stored product pests are of- 
ten only released when males are on suitable breeding substrate. However, in our study, using a four-zone bioassay chamber, results from experiment 3 showed that the aggregation pheromone is released by males within about 13 minutes after removal from the grains. However, the release of aggregation pheromone by males is stopped about 18 minute after.

As stated above, our results suggest that the release of aggregation pheromone by $S$. zeamais males after removal from the grains phase out with the time going by.

\subsection{Enhancement of attraction responses to aggregation pheromone by host odour}

Male produced aggregation pheromones may be considered the most important stimuli for hostplant location in stored-product beetles and play a crucial role in subsequent aggregation by conspecific male and female.

Odours from larval foods or host plants are known to synergistically enhance the responses of phytophagous insects to their pheromones (Wood 1982, Dickens et al. 1990). Interactions among pheromones and host-plant volatiles have been the subject of several recent reviews (Landolt 1997, Landolt \& Phillips 1997, Reddy \& Guerrero 2004). A strong source of host volatiles combined with aggregation pheromone, in amounts naturally produced by males, substantially increases the attraction responses of $S$. zeamais.

Very little is known about the mechanism of how host-plant chemistry would affect attractiveness of pheromone signals in S. zeamais. It might be thought that the increased attraction to the host volatile combined with aggregation pheromone have two possible reasons. One is a function of the host volatiles directly. The other is an increased pheromone output by males in response to their proximity to a large food supply $(500 \mathrm{~g}$ wheat). In a laboratory study, Bashir et al. (2003) showed that the absolute quantities of the aggregation pheromones (DL-1 or DL-2) produced were lowered differentially when male $R$. dominica were moved from suitable host to unsuitable host materials, but that pheromone release rate and ratio were restored when the move was re- versed. Apparently, males respond by emitting less pheromone on unsuitable host, making it less likely that migrant conspecifics will detect the pheromones (Roelofs 1978, Baker \& Kuenen 1982, Byers 1995).

Optimal use of pheromones for insect management may depend on knowledge of interactions among pheromones and host-plant volatiles (Landolt \& Phillips 1997).

\subsection{Difference in response to infochemicals between males and females}

A previous investigation of the orientation response of male and female $S$. zeamais to semiochemicals suggested that the response of the two sexes was similar (Ben \& Xuan 1992). However, in our study, using a four-zone bioassay chamber, results obtained from experiment 3 and experiment 4 showed clear differences between male and female responses. In the multiple-choice tests, when beetles could select between host volatiles alone or the males alone or host volatile combined pheromone sources, females spent hardly any time in the zone receiving host volatiles alone (on average $7.9 \%$ ). In contrast, males spent significantly more time in the zone with host volatiles (on average $26.2 \%$ ), which shows that males are relatively more attracted than females to host odours and suggest that they may be more highly adapted to seek out new hosts from which they can attract mates while females are more inclined to locate a suitable food source by following the pheromone signals produced by males.

The results also showed that host volatiles were relatively less attractive for female in the presence of aggregation pheromones and their responses might be largely mediated by aggregation pheromone. Females were significantly more attracted than males to the host volatile combined pheromone source, confirming the stronger response of females to pheromone.

The observation could reflect a difference between males and females in the strength of response to the pheromone, in which females are simply more sensitive; it could also reflect differences in search behaviour strategies by the sexes, in which males may remain at a distance to avoid 
competition with other males and females driven to find host material for oviposition which has been located by males. This is consistent with the previous report in which sexual selection is stronger on males than females so that males would be expected to take greater reproductive risks (Alcock \& Gwynne 1991), in this case finding a new host in isolation.

Females spent more time (on average $71.3 \%$ ) in the zone of host volatile combined pheromone source than males (on average 55.1\%). Therefore, it is not surprisingly that no significant differences were observed that females spent time (on average 19.2\%) in the zone of pheromone alone source than males (on average 15.4\%). The observations support the view that both males and females respond to host volatiles and pheromone and more strongly to the mixture of the two.

Acknowledgements. We are grateful to Professor Jiawei Du (Institute of Plant Physiology and Ecology, Shanghai Institutes for Biological Sciences, Chinese Academy of Sciences) and Professor Le Kang (State Key Laboratory of Integrated Management of Pest Insects and Rodents, Institute of Zoology, Chinese Academy of Sciences) for valuable suggestions during this study. We thank Dr. Yansheng Zhang (Natural Product Department, The Plant Biotechnology Institute, National Research Council of Canada) for modification and comments on the manuscript. The research was financially supported by the Knowledge Innovative Program of the Chinese Academy of Sciences, Grant No.: KSCX-SW-324.

\section{References}

Adane, K., Moore, D. \&, S. A. 1996: Preliminary studies on the use of Beauveria bassiana to control Sitophilus zeamais (Coleoptera: Curculionidae) in the laboratory. - Journal of Stored Product Research 32: 105-113.

Alcock, J. \& Gwynne, D. T. 1991: Sexual selection and the evolution of mating systems. - In: W. J. Bailey \& J. Riddill-Smith (eds.), Reproductive Behaviour in Insects: 10-41. Chapman \& Hall, London.

Baker, T. C. \& Kuenen, L. P. S. 1982: Pheromone source location by flying moth: a supplementary non-anemotactic mechanism. - Science 216: 424-427.

Bashir, T., Birkinshaw, D. R. \& Hodges, R. J. 2001: Host odours enhance the responses of adult Rhyzopertha dominicato male-produced aggregation pheromone. - Entomologia Expermentalis et Applicata 101: 273>@150<280.

Bashir, T., Hodges, R. J., Birkinshaw, L. A., Hall, D. R. \& Farman D. I. 2003: Phenotypic plasticity of Rhyzopertha dominica pheromone signaling: the effects of different hosts and presence of conspecific females on male produced aggregation pheromone. - Journal of Chemical Ecology 29: 945-959.

Ben, T. B. \& Xuan, J. Y. 1992: (Chemical ecology of coleopterous stored-product pests and its applications). Plant Doctor 5: 17-19,31. [In Chinese.]

Byers, J. A. 1995: Host tree chemistry affecting colonization in bark beetles. - In: Cardé R. T. \& Bell W. J. (eds.), Chemical Ecology of Insects (2nd Edition): 154-213. Chapman \& Hall, New York, NY, USA.

Dickens, J. C., Jang, E. B., Light, D. M. \& Alford, A. R. 1990: Enhancement of insect pheromone responses by green leaf volatiles. - Naturwissenschaften 77: 2931 .

Dobie, P. 1974: The laboratory assessment of the inherent susceptibility of maize varieties to post-harvest infestation by Sitophilus zeamais Motsch. (Coleoptera, Curculionidae). - Journal of Stored Product Research 10: 183-197.

Edde, P. A. \& Phillips, T. W. 2006: Potential host affinities for the lesser grain borer, Rhyzopertha dominica: behavioral responses to host odours and pheromones and reproductive ability on non-grain hosts. - Entomologia Expermentalis et Applicata 119: 255-263.

Faruki, S. I., Miyanoshita, A. \& Takahashi, K. 2005: Susceptibility of various developmental stages of the maize weevil, Sitophilus zeamais Motschulsky (Col., Curculionidae) to methyl iodide in brown rice. Journal of Applied Entomology 129: 12-16.

Francis, F., Vandermoten, S., Verheggen, F., Lognay, G. \& Haubruge, E. 2005: Is the (E)- $b$-farnesene only volatile terpenoid in aphids? - Journal of Applied Entomology 129: 6-11.

Giles, P. H. 1969: Observations in Kenya on flight activity of stored products insects particularly Sitophilus zeamais Motsch. - Journal of Stored Product Research 4: 317-329.

Gudrups, I., Flovd, S., Kling, J. G., Bosque-Perez, N. A. \& Orchard, J. E. 2001: A comparison of two methods of assessment of maize varietal resistance to the maize weevil, Sitophilus zeamais Motschulsky, and the influence of kernel hardness and size on susceptibility. - Journal of Stored Product Research 37: 287-302.

Haines, C. P. 1991: Insects and Arachnids of Tropical Stored Products: Their Biology and Identification, 2nd edition. Natural Resources Institute, UK. 246 pp.

Halstead, D. G. H. 1963: The separation of Sitophilus oryzae (L.) and S. zeamais Motschulsky (Col., Curculionidae), with a summary of their distribution. - Entomologist's Monthly Magazine 99: 72-74.

Hill, D. S. 1983: Agricultural insect Pests of the Tropics and their Control. 2nd edition. Cambridge University Press, Cambridge. 146 pp.

Hodges, R. J. \& Dobson, C. C. 1998: Laboratory studies on behavioural interactions of Prostephanus truncatus (Horn) (Coleoptera: Bostrichidae) with conspecifics, synthetic pheromone and the predator Teretriosoma nigrescens (Lewes) (Coleoptera: Histeridae). — Journal of Stored Product Research 34: 297-305.

Honda, H. \& Oshawa, O. 1990: Chemical ecology for sto- 
red product insects. - Journal of Pesticide Science 15: 263-270.

Kouninki, H., Hance1, T. \& Noudjou F. A. : Toxicity of some terpenoids of essential oils of Xylopia aethiopicafrom Cameroon against Sitophilus zeamaisMotschulsky. - Journal of Applied Entomology 131: 269-274.

Landolt, P. J. 1997: Sex attractant and aggregation pheromones of male phytophagous insects. - American Entomologist 43: 12-22.

Landolt, P. J., \& Phillips, T. W. 1997: Host plant influences on sex pheromone behavior of phytophagous insects. - Annual Review of Entomology 42: 371-391.

Negahban, M. \&Moharramipour, S. 2007: Fumigant toxicity of Eucalyptus intertexta, Eucalyptus sargentii and Eucalyptus camaldulensis against stored-product beetles. - Journal of Applied Entomology 131: 256261.

Pike, V., Smith, R. D. \&Hall, D. R. 1994: Studis of reponses of stored-product pests, Prostephanus truncatus (Horn) and Sitophilus zeamais., to food volatiles. In: Highley, E., Wright, E. J., Banks, H. J. \&Champ, B. R. (eds.), of the 6thWorking Conference on Stored Products Protection, Canberra, Australia, 17-22 April 1994, Vol. 1: 566-568.

Plarre, R. 1998: Pheromones and other semiochemicals of stored product insects - A historical review, current application, and perspective needs. - Mitteilungen aus der Biologischen Bundesanstalt für Land- und Forstwirtschaft 342: 13-83.

Povey, S. R. \& Sibly, R. H. 1992: No oviposition plasticity in Sitophilus oryzae. - Journal of Stored Product Research 28: 11-14.

Pureswaran, D. S. \& Borden, J. H. 2005: Primary attraction and kairomonal host discrimination in three species of Dendroctonus (Coleoptera: Scolytidae). - Agricultural and Forest Entomology 16: 765-782.

Reddy, G. V. P. \& Guerrero, A. 2004: Interactions of insect pheromones and plant semiochemicals. - Trends in Plant Science 9: 253-261.

Roelofs, W. L. 1978: Threshold hypothesis for pheromone perception. - Journal of Chemical Ecology 4: 685699.
SAS Institute 2001: SAS/STAT User's Guide, Version 8. - SAS Institute Inc., Cary, NC, USA.

Storey, C. L. 1987: Effect and control of insects affecting corn quality. - In: Watson, S. A., Ramstad, P. E. (eds.), Corn Chemistry and Technology: 185-199. American Association of Cereal Chemists, St. Paul.

Takabayashi, T. \& Dicke, M. 1992: Response of predatory mites with different rearing histories to volatiles of uninfested plants. - Entomologia Expermentalis et Applicata 64: 187-193.

Trematerra, P. \& Girgenti, P. 1989: Influence of pheromone and food attractants on trapping of Sitophilus oryzae (L.) (Coleoptera: Curculionidae): a new trap. Journal of Applied Entomology 108: 12-20.

Tukey, J. W. 1953: The Problem of Multiple Comparisons. - Department of Statistics, Princeton University, Princeton, NJ, USA.

Vet, L. E. M., Van, Lenteren, J. C., Heymans, M. \& Meelis, E. 1983: An airflow olfactometer for measuring olfactory responses of hymenopterous parasitoids and other small insects. - Entomology 8: 97-106.

Visser, J. H. 1986: Host odour perception in phytophagous insects. - Annual Review of Entomology 31: 121144.

Walgenbach, C. A. \& Burkholder, W. E. 1987: Mating behaviour of the maize weevil Sitophilus zeamais (Coleoptera: Curculionidae). — of the Entomolgical Society of America 80: 578-583.

Walgenbach, C. A., Phillips, J. K., Faustini, D. L. \& Burkholder, W. E. 1983: Male-produced aggregation pheromone of the maize weevil, Sitophilus zeamais, and interspecific attraction between three Sitophilus species. - Journal of Chemical Ecology 9: 831-841.

Walgenbach, C. A., Burkholder, W. E., Curtis, M. J. \& Khan, Z. A. 1987: Laboratory trapping studies with $\mathrm{Si}$ tophilus zeamais (Coleoptera: Curculionidae). Journal of Economic Entomology 80: 763-767.

Wood, D. L. 1982: The role of pheromones, kairomones, and allomones in the host selection and colonization behavior of bark beetles. - Review of Entomology 27: 411-446.

Zar, J. H. 1999: Biostatistical Analysis. — Prentice Hall, Inc., Inglewood Cliffs, New Jersey. 